\title{
The role of primary care physicians in early diagnosis and treatment of chronic gastrointestinal diseases
}

This article was published in the following Dove Press journal:

International Journal of General Medicine

13 March 2014

Number of times this article has been viewed

\author{
Aristofanis Gikas' \\ John K Triantafillidis ${ }^{2}$ \\ 'Health Center of Kalivia, Kalivia, \\ Attiki, Greece; ${ }^{2}$ Inflammatory Bowel \\ Disease Unit, IASO General Hospital, \\ Athens, Greece
}

\begin{abstract}
Chronic gastrointestinal disorders are a source of substantial morbidity, mortality, and cost. They are common in general practice, and the primary care physician (PCP) has a central role in the early detection and management of these problems. The need to make costeffective diagnostic and treatment decisions, avoid unnecessary investigation and referral, provide long-term effective control of symptoms, and minimize the risk of complications constitute the main challenges that PCPs face. The literature review shows that, although best practice standards are available, a considerable number of PCPs do not routinely follow them. Low rates of colorectal cancer screening, suboptimal testing and treatment of Helicobacter pylori infection, inappropriate use of proton pump inhibitors, and the fact that most PCPs are still approaching the irritable bowel disease as a diagnosis of exclusion represent the main gaps between evidencebased guidelines and clinical practice. This manuscript points out that updating of knowledge and skills of PCPs via continuing medical education is the only way for better adherence with standards and improving quality of care for patients with gastrointestinal diseases.
\end{abstract}

Keywords: guidelines, screening, treatment

\section{Introduction}

Gastrointestinal (GI) diseases are a source of substantial morbidity, mortality, and cost. Spending on GI diseases in the US has been estimated at $\$ 142$ billion per year in direct and indirect costs, with the total cost for outpatient GI endoscopy estimated at $\$ 32.4$ billion. ${ }^{1}$ GI disorders are common in general practice, accounting for about $10 \%$ of the work of general practitioners. ${ }^{2}$ In the era of rapid change in the health care environment and with an emphasis on cost containment, primary care physicians (PCPs) face specific challenges in their management, for example, of the cost effective treatment of dyspepsia and gastroesophageal reflux, the skillful analysis of GI symptoms, and the need for early detection of cancer. Furthermore, the close relationships that digestive disorders have with lifestyle habits, and their huge impact on quality of life, requires a holistic approach with attention not only to organic but also to psychosocial aspects of GI diseases.

During the last few years, the role of PCPs in the diagnosis and management of GI disorders has been recognized as very important, and it has been suggested that they have all the available resources in order to ensure high standard of care for their patients. In particular, clearly articulated clinical practice guidelines, effective medications, accurate noninvasive investigations, and evidence-based primary care management plans are available to support PCPs who want to raise their threshold for referring patients with GI symptoms. ${ }^{3}$
Correspondence: Aristofanis Gikas

Themistokleous 17, Maroussi,

15122, Greece

$\mathrm{Tel}+302121025094$

Fax +302299048706

Email argikas@ath.forthnet.gr submit your manuscript $\mid$ www. dovepress.com

Dovepress

http://dx.doi.org// 0.2147/IJGM.S58888
International Journal of General Medicine 2014:7 I59-I73

159

cc) (i) (5) 2014 Gikas and Triantafilidis. This work is published by Dove Medical Press Limited, and licensed under Creative Commons Attribution - Non Commercial

(c) (i) (unported, v3.0) License. The full terms of the License are available at http://creativecommons.org/licenses/by-nc/3.0/. Non-commercial uses of the work are permitted without any further permission from Dove Medical Press Limited, provided the work is properly attributed. Permissions beyond the scope of the License are administered by Dove Medical Press Limited. Information on how to request permission may be found at: http://www.dovepress.com/permissions.php 
In this narrative review, we explore the published literature on the existing and potential roles of the PCP in the diagnosis and management of GI disorders. The relevant evidence was found through literature searches on Medline, PubMed, and guidelines. Articles reviewed were identified from keywords related with primary care, best practice standards, guidelines implementation, and specific GI disorders. Focusing on most common chronic GI diseases (common acute disorders such as gastritis and gastroenteritis are not addressed in this article), we highlight the relevant medical standards and provide the available data concerning practices, level of awareness, and implementation of guidelines among PCPs. Moreover, special attention was given to the differences between PCPs and gastroenterologists (GEs) in diagnosing and managing GI disorders. Given the growing role of PCPs, the continuous advances in technology, and the changes in epidemiology of GI diseases, we underscore the ways to update PCP awareness and implementation of the evidence based guidelines.

\section{The role of PCPs in early diagnosis of malignant $\mathrm{GI}$ disorders Colorectal cancer}

Colorectal cancer (CRC) represents one of the most important malignant digestive diseases harboring significant morbidity and mortality. Early detection (Dukes' A and B) represents the only chance for increasing the rate of 5-year survival. On the other hand, as the great majority of CRC appears in the context of preexisting colorectal polyps, early endoscopic detection and removal of colorectal polyps diminishes the incidence of this neoplasm in countries where programs on the prevention of CRC are applied. Guidelines recommend that all men and women be screened for CRC beginning at age 50 years (or earlier if they are at increased risk because of a family history of CRC), and it has been generally accepted that successful screening starts with primary care. ${ }^{4}$ However, a large part of the population does not follow the guidelines provided by the relevant scientific organizations and country health authorities. In the US, for example, half of the population aged over 50 years does not participate in prevention programs for CRC. An easier access to centers performing the necessary examinations and better population education might have a positive impact on the rate of people participating in these programs. ${ }^{5}$ PCPs could play an essential role in persuading people to participate in these programs and in supporting families with members suffering from CRC. ${ }^{6}$

A practical question is: are the guidelines published by the international scientific organizations familiar to PCPs and, if so, are they applied correctly? In a relevant study conducted in Israel, it was found that the suggestion for annual fecal occult blood test (FOBt) was correctly performed by $40 \%$ of PCPs, the suggestion for flexible sigmoidoscopy every 3 to 5 years by $12 \%$, and the concurrent application of both examinations by $8 \%{ }^{7}$ In Greece, only $50 \%$ of PCPs recommend screening for CRC; the percentage of PCPs recommending FOBt and sigmoidoscopy was $24 \%$ and $4 \%$, respectively. ${ }^{8}$

A review from Mauri et al reported that, in other European countries such as France and Italy, CRC screening was recommended by $65 \%-95 \%$ of physicians; FOBt was advised by $42 \%-83 \%$ and prescription of screening endoscopic modalities was inconsistent $(6 \%-48 \%) .{ }^{9}$ Based on these data, it has been suggested that, in comparison with European practice, CRC screening habits of US physicians are to a greater extent rational, evidence-based, well monitored, and have a longer tradition in medical care, thus allowing better prevention services for asymptomatic individuals. It is likely that those differences explain the findings of a recent study that showed that patients diagnosed at later stages (Dukes' D and advanced stage) were less common in the US than in the four European regions (especially in southern and eastern European countries). ${ }^{10}$ Moreover, taken together, these data may explain the wide differences in CRC survival between Europe and the US in the late 1990s.

It seems that the personal involvement of PCPs has a positive impact on the participation of people in screening programs for CRC. In a study in Australia, letters calling for participation in CRC screening programs have better results if they are accompanied by the personal involvement of PCPs. ${ }^{11}$ In a recent study, it was found that participants who receive the FOBt kit from their PCP are more likely to participate in the study. ${ }^{12}$

The majority of patients with CRC seek medical advice from PCPs in cases in which relevant symptoms are present. It is, therefore, crucial to evaluate the most important symptoms (change in bowel habit, rectal bleeding, anemia, etc) that could lead the PCP to correctly diagnose the underlying CRC. In a study in Italy, it was noticed that only two factors were significantly related with the presence of CRC; namely, an age greater than 50 years and iron-deficiency anemia. ${ }^{13}$ The findings of a systematic review suggest that investigation of rectal bleeding or anemia in primary care patients is warranted, irrespective of whether other symptoms are present. The risks from other single symptoms are lower, though multiple symptoms also warrant investigation. ${ }^{14}$

Colonoscopy remains the gold standard for the investigation and management of bowel pathology. Due to the very low 
number of endoscopists, it is impossible even for more developed health systems to enact a CRC screening program with colonoscopy. PCPs could play an important role in filling this shortage by offering screening colonoscopy in their practice. It is obvious that the prerequisite for accomplishment of this task is suitable training of PCPs - a goal that harbors many difficulties in most countries. ${ }^{15}$ In 2002, there were 8,207 endoscopy centers in the US able to perform sigmoidoscopy and colonoscopy. GEs performed 44\% of rectosigmoidoscopies and $83 \%$ of colonoscopies, while PCPs performed $25 \%$ of sigmoidoscopies and $2 \%$ of colonoscopies. In total, 2.8 million sigmoidoscopies and 14.2 million colonoscopies were performed. The available personnel could perform 9.5 million sigmoidoscopies and 22.4 million colonoscopies annually, suggesting that, with active involvement of PCPs, the whole population of the US could be efficiently screened for CRC. ${ }^{16}$ Data from different settings in different countries shows that adequately trained family physicians can provide safe and technically competent colonoscopy. Their results compare favorably to the currently reported comparative benchmarks from other endoscopists. ${ }^{17-20}$

\section{Gastric cancer}

Gastric cancer (GC) is the second most common cause of death worldwide in all types of malignant tumors, preceded only by lung cancer. Early detection and treatment is the only way to reduce mortality. At present, no consensus exists for the GC screening program. Some countries of the East Asia region such as Japan and South Korea, which have a high incidence of GC, have achieved tangible results from population-based screening strategies. Barium double-contrast radiography combined with endoscopy is a mature method that has been used in Japan for over 60 years. ${ }^{21}$ A new strategy using simultaneous measurement of serum pepsinogens and Helicobacter pylori antibody combined with eradication of $H$. pylori in all individuals at risk has been proposed. ${ }^{22}$ No nationwide screening of GC has been reported in the US, Europe, and other areas with a low GC incidence; it seems that adoption of GC screening in moderate- to high-risk population subgroups constitutes a cost-effective and feasible strategy. ${ }^{23}$ Thus, the identification of subjects with high risk factors (family history of GC, smoking, alcohol abuse, previous stomach surgery, $H$. pylori infection, salted and smoked food intake) by PCPs should be part of their routine clinical practice.

A significant proportion of patients with early GC experience nonspecific dyspeptic symptoms. Because dyspepsia is very common in the general population, the difficulty confronting PCPs is in how to separate the wheat from the chaff in deciding which patients should be referred early for investigation. ${ }^{24}$ Given the fact that gastric malignancy is rare before age 40 years, and the incidence increases steadily thereafter, referral for endoscopy is recommended for all patients aged over 45 with new onset dyspepsia. Moreover, alarm symptoms such as weight loss, dysphagia, signs and symptoms of upper GI bleeding, anemia, and persistent vomiting are likely to be more frequently associated with upper GI malignancies, and most guidelines recommend immediate endoscopy in all patients presenting with these symptoms. ${ }^{25}$

Although guidelines recommend that upper and lower GI investigations should be considered in all postmenopausal female and all male patients with iron-deficient anemia, ${ }^{26} \mathrm{GI}$ investigations seem to be performed suboptimally by PCPs. In a study in the UK, it was noticed that $47 \%$ of 431 patients presenting to their general practitioner with an iron-deficient anemia were adequately managed and $39 \%$ of patients who were otherwise fit for investigation had no tests at all. It is worth noticing that only 29 of the 41 GI cancers (22 lower, seven upper) were found as a result of satisfactory GI investigations. ${ }^{27} \mathrm{~A}$ similar study from the Netherlands showed that only $31 \%$ of male and postmenopausal female patients received some form of endoscopic evaluation. ${ }^{28}$

During the management of suspicious cases for upper GI malignancy, PCPs must bear in mind that treatment of dyspeptic symptoms with acid suppression therapy prior to gastroscopy masks and delays the detection of gastric and esophageal adenocarcinoma on endoscopy. ${ }^{29,30}$ There is endoscopic evidence showing that early malignancy within the gastric mucosa may be healed with acid suppression therapy, particularly proton pump inhibitors (PPIs). Thus, PCPs must not hastily prescribe PPIs before endoscopy, particularly in patients older than 45 years. ${ }^{31}$

Experience from countries with a more developed health system has shown that open-access gastroscopy performed by general practitioners at primary care health centers is effective and, as a result, the subsequent GI consultations become less frequent and the level of compliance with endoscopist recommendations becomes higher. Moreover, a relevant study from Finland showed that no significant difference was detected in the outcome of patients diagnosed in primary care centers by general practitioner endoscopists compared to in hospital outpatient clinic by specialists. ${ }^{32}$

\section{Esophageal cancer}

Esophageal cancer (EC) is a common malignancy with a very poor prognosis. In contrast with the worldwide decrease in the 
incidence of GC, which may be attributed to the aggressive treatment of $H$. pylori, it seems that the overall incidence of EC is rising. The two main risk factors for esophageal adenocarcinoma are gastroesophageal reflux and obesity, and for squamous cell carcinoma of the esophagus the main risk factors are smoking and high alcohol consumption, particularly in combination. ${ }^{33}$

Progressive dysphagia or odynophagia and weight loss are the most common presenting complaints of patients with EC. Patients presenting with those symptoms should undergo urgent endoscopy, preferably within 1 week. Evidence shows that diagnosis of EC is often delayed, and the interval between symptom onset and diagnosis ranges from 1-11 months. ${ }^{34,35}$ Given the limited usefulness of individual symptoms (eg, dysphagia) for diagnosis, a more appropriate approach focused on a combination of multiple risk factors is needed. Recent studies have developed and validated multivariable prediction models that can be used to identify patients with an existing but as yet undiagnosed gastroesophageal cancer. ${ }^{36,37}$ It is suggested that these new models can help PCPs to identify those at highest risk of gastroesophageal cancer in order to facilitate early referral and investigation and so to minimize delay in diagnosis.

There is a proven association between adenocarcinoma and Barrett's esophagus - a condition in which metaplastic columnar epithelium replaces normal stratified squamous mucosa that appears to arise in response to chronic inflammation from gastroesophageal reflux disease (GERD). It is estimated that about 1 in 300 patients with Barrett's esophagus will develop EC each year. ${ }^{38}$ Current guidelines recommend that endoscopic screening for Barrett's esophagus can be considered in patients with chronic GERD symptoms and multiple risk factors (at least three of: age 50 years or older, white race, male sex, obesity). However, the threshold of multiple risk factors should be lowered in the presence of family history including at least one first-degree relative with adenocarcinoma and Barrett's esophagus. ${ }^{38}$

\section{The role of PCPs in early diagnosis and management of patients with benign $\mathbf{G I}$ disorders Dyspepsia, peptic ulcer, and $H$. pylori}

Dyspepsia is a common clinical problem with about one fifth of people affected at some point in their lives. ${ }^{39}$ The management of dyspepsia represents a large component of clinical practice in primary care, accounting for $5 \%$ of all general practice consultations. ${ }^{40}$ Around $70 \%-80 \%$ of patients with dyspeptic symptoms will have functional dyspepsia where no underlying cause is detected at endoscopy. A systematic review that analyzed nine studies involving more than 5,000 people with dyspepsia showed that there was a $13 \%$ prevalence of esophagitis and $8 \%$ prevalence of peptic ulcer disease, with gastric or EC occurring in less than $0.3 \%$ of endoscopies. ${ }^{41}$ Esophagitis was more prevalent in Western populations than in Asian ones (25\% versus 3\%), whereas the opposite was true for peptic ulcer disease (3\% versus $11 \%$ ) differences which probably reflect variations in the prevalence of $H$. pylori infection. Data show that dyspeptic symptoms are not a good predictor of the underlying endoscopic findings; however, guidelines recommend that patients with alarm symptoms should be referred for upper GI endoscopy examination, as these may be suggestive of a malignancy.

Taking into account this evidence, in regions where the prevalence of $H$. pylori infection is $>20 \%$, current management strategies emphasize testing and treatment for H. pylori in the initial management of patients presenting with dyspeptic symptoms. ${ }^{42}$ If the prevalence of $H$. pylori infection in a specific population is low, it makes sense to use an acid suppression strategy. Although peptic ulcer disease has declined in Western countries, specific populations such as immigrants and rural communities may have a high prevalence of infection and peptic ulcer disease that needs to be considered in dyspepsia management, even in areas where the prevalence of $H$. pylori infection has declined to below $15 \% .{ }^{43}$ Therefore, in circumstances of changing epidemiology of dyspepsia and underlying disease, it is important for PCPs to have the knowledge of approximate prevalence of $H$. pylori infection in their respective communities. In patients who are symptomatic without an organic pathology, functional dyspepsia and other causes of abdominal pain need to be considered. Functional dyspepsia is best managed using a multifaceted approach by establishing a good physicianpatient relationship, dietary and lifestyle interventions, acid suppression therapy, psychotherapy, and the use of psychotropic medications. ${ }^{44}$

The best-known guidelines regarding dyspepsia and H. pylori infection have been disseminated for more than 15 years $^{45,46}$ and it is of interest to take a glimpse in the available literature concerning awareness of and guidelines for implementation by PCPs. A study conducted in Ireland showed that only $60 \%$ of the $H$. pylori-positive patients seemed to receive appropriate eradication therapy, and almost a quarter appeared to receive no prescription. Of those with a positive urea breath test who received eradication therapy, only $43 \%$ were referred back for retesting. This percentage 
is low, particularly as confirmation of successful eradication is recommended by current guidelines. Moreover, the study showed that a significant proportion of the examined population were aged 45 years and over (54\%) and, if recommended guidelines were adhered to, this group should have undergone endoscopy to investigate their dyspepsia rather than a test-and-treat approach ${ }^{47}$ Another study conducted in Korea showed that about $65 \%$ of PCPs tried to eradicate H. pylori-positive cases in patients with dyspepsia, and only $9 \%$ recommended a follow-up testing. ${ }^{48}$ As shown by Ahmed et al, with regard to Pakistani PCPs' lack of knowledge regarding management of $H$. pylori infection, only $35 \%$ of the physicians were of the view that urea breath test was the most appropriate test to diagnose active $H$. pylori infection, and $77 \%, 64 \%$, and $19 \%$ of them thought that gastric ulcer, duodenal ulcer, and mucosa-associated lymphoid tissue lymphoma was the most compelling indication for treatment, respectively. ${ }^{49}$

A Pan-European survey demonstrated substantial variations between general practitioners in management of dyspepsia. ${ }^{50}$ In particular, test and treat for $H$. pylori was frequently used as an initial management strategy in England (47\%), Poland (40\%), and Greece (32\%), but less commonly elsewhere. Early endoscopy as first choice management of dyspepsia was more likely to be selected in the Czech Republic (32\%) and Greece (20\%) than in other countries. In the treatment of nonulcer dyspepsia, $H$. pylori infection was used by $90 \%$ of the UK respondents, $82 \%$ in Greece, $72 \%$ in Spain, $71 \%$ in the Netherlands, $64 \%$ in Poland, and only $30 \%$ in the Czech Republic.

A 2002 report from O'Connor ${ }^{51}$ describes that, in surveys conducted in the early 1990s, significantly more GEs than PCPs believed that $H$. pylori was causal in duodenal ulcer; however, in later surveys, this gap had narrowed to the point where almost all PCPs and GIs believed that $H$. pylori was causal in duodenal ulcer. ${ }^{51}$ This trend is also apparent in attitudes toward the prescription of $H$. pylori therapy for duodenal ulcer. In the early 1990s, H. pylori therapy for duodenal ulcer was prescribed significantly more often by GEs than PCPs and, as Hirth et $\mathrm{al}^{52}$ demonstrated, GEs adopted $H$. pylori therapy for duodenal ulcer approximately 21 months earlier than PCPs. Moreover, the aforementioned report ${ }^{51}$ showed that $H$. pylori therapy for nonulcer dyspepsia was prescribed by $41 \%-66 \%$ of PCPs compared with $27 \%-43 \%$ of GEs, and this prescription pattern showed no significant change with time.

A 2009 study by Spiegel et al ${ }^{53}$ found that there was a significant difference in guideline adherence regarding dyspepsia between GEs and PCPs (74\% versus 57\%, respectively). Moreover, they found that PCPs were more likely to define dyspepsia incorrectly, to perform nonguideline-supported diagnostic testing (eg, abdominal ultrasound, radiography, computerized tomography), to test for $H$. pylori with serology, delay endoscopy in patients $>55$ years old, and to avoid first-line PPIs in lieu of other medical therapies. The study also shows that PCPs were more concerned than GEs for potential adverse effects of long-term PPI therapy. The highest concerns were registered for osteoporosis, community-acquired pneumonia, and vitamin B12 deficiency.

PPIs constitute the mainstay therapy for upper GI disorders and are one of the most frequently prescribed classes of medications in the world. Absolute indications include dyspepsia, peptic ulcer disease, treatment of H. pylori, chronic nonsteroidal anti-inflammatory-drug (NSAID) use, and GERD. ${ }^{54}$ Despite proper guidelines, there is growing concern about the exponential increase and inappropriate prescription of PPI therapy in primary care. ${ }^{55-58}$ It was observed that a considerable proportion (36\%-54\%) of patients taking PPIs were prescribed PPIs for an indication outside those proposed in current guidelines. This poses economic and safety concerns, particularly in light of the suggestion that these drugs could delay the diagnosis of GC.

The rationale for use of PPIs for gastroprotection constitutes an important issue in routine practice of PCPs. The international guidelines recommend the use of gastroprotective therapy (with PPIs being the preferred agents) for at-risk patients taking any NSAID; advanced age ( $>65$ years), a personal history of peptic ulcer, the presence of serious comorbidities, and concomitant treatment with either anticoagulants, corticosteroids, or other NSAIDs have been identified as significant risk factors for gastrointestinal events during NSAID therapy. Testing for and eradication of H. pylori in patients at high risk of NSAID-related gastrointestinal bleeding should be considered, but will be insufficient without ongoing gastroprotection..$^{59} \mathrm{~A}$ recent survey assessing the appropriateness of PCPs, management of gastroprotective therapy in NSAID users showed a disappointing $66 \%$ rate of inappropriate (overuse/underuse) indication, suggesting that roughly only one out of every three NSAID users may be expected to leave the PCP office with the appropriate management. ${ }^{60,61}$ Data on $H$. pylori-infection management showed that the $H$. pylori infection status was investigated in $16 \%$ of patients receiving chronic NSAID therapy, and it was eventually cured in $73 \%$ of the infected cases. ${ }^{60,61}$ 


\section{Gastroesophageal reflux disease}

GERD is a highly prevalent condition defined as symptoms or complications resulting from the reflux of gastric contents into the esophagus, or beyond into the oral cavity (including larynx) or lung. Epidemiological evidence indicates that the prevalence of GERD in the Western world is $10 \%-20 \%$, with a lower prevalence in Asia. ${ }^{62}$ GERD represents the fourth most common chronic condition - after hypertension, hyperlipidemia, and depression - seen in primary care practice ${ }^{63}$ Because of its high prevalence, care of patients with GERD is largely within the domain of PCPs. The early released as well as updated GERD guidelines promote history taking as the most useful method of diagnosis. ${ }^{62,64}$ The symptoms of heartburn and regurgitation are the most reliable for making a presumptive diagnosis based on history alone; however, these symptoms are not as sensitive as most believe. It is also recommended that empiric PPI therapy (including lifestyle modification) is a reasonable approach to confirm GERD when it is suspected in patients with typical symptoms. Patients with GERD may present with a broad range of troublesome symptoms, beyond heartburn and regurgitation, which can overlap with other GI diseases (such as dyspepsia) and may include chest pain or extraesophageal manifestations, such as chronic cough and asthma. Caution is needed for patients with chest pain; a cardiac cause should be excluded before the commencement of a GI evaluation.

Many patients with typical GERD symptoms do not have endoscopic erosive disease, suggesting that endoscopy is of limited value in guiding disease management. Endoscopy is recommended if the patient does not respond in 4-8 weeks of twice daily PPI therapy, in the presence of alarm symptoms (dysphagia, odynophagia, bleeding, anemia, weight loss, or recurrent vomiting), and for screening of patients at high risk for Barrett's esophagus (age over 50 years, symptoms for $>5$ years, obesity, male sex).

Despite the prevalence and impact of GERD, along with the availability of effective treatments on prescription, a considerable number of subjects with symptoms suggestive of GERD have inadequate disease management. Jones et al, ${ }^{65}$ based on data from a multinational survey, reported that $78 \%$ of diagnosed subjects were currently receiving medication prescribed by their doctor, and $65 \%$ were taking over-thecounter treatments. Despite medication, $58 \%$ of diagnosed and $73 \%$ of undiagnosed subjects still experienced GERD symptoms some of the time. In addition, approximately one third of subjects reported that they ate less than usual, felt generally unwell, were tired/worn out, or were worried/ fearful for the majority of the time because of their GERD symptoms, and around half reported decreased well-being, including reduced work or leisure time productivity. A recent Pan-European study showed that $30 \%-100 \%$ of patients were prescribed a PPI, but a significant GERD-symptom load was still experienced by $15 \%-30 \%$ of patients at follow-up (median 5.0-7.5 months after initial consultation). ${ }^{66}$

More data that provide insight into the ways of management of GERD patients come from physician-based surveys. A relevant Pan-European study showed that the majority of PCPs (varying from $51 \%$ in the UK to $84 \%$ in Czech Republic) were aware of the test with PPI as a method of diagnosing GERD, but the daily PPI dose used by them for this test ranged from maintenance dose to fourfold standard treatment dose, over a period that ranged from 1 to 13 weeks. ${ }^{50}$ Earlier studies showed that PCPs preferred a step-up strategy beginning with antacids or H2-receptor antagonists and progressing to PPIs for patients who fail to respond to therapy, ${ }^{67,68}$ while the more recent studies show that the majority of PCPs tend to begin with the most efficacious therapy with PPIs and then taper the dose down to the lowest dose that controls symptoms (stepdown strategy). ${ }^{69,70}$ In addition, GEs are uniformly likely to use a step-down therapy regimen.

Bretagne et al, ${ }^{69}$ when comparing PCPs and GEs, observed that alarm symptoms were identified more frequently by PCPs than GEs, but the appraisal of their seriousness was less acute by PCPs than GEs. Upper endoscopy was prescribed more frequently by GEs compared to PCPs (64\% versus $38 \%$, $P<0.01$ ), suggesting that GEs tend to use a more resourceintensive approach to GERD, performing diagnostic procedures before commencing a treatment regimen. Data from this survey show that a number of lifestyle changes were almost constantly recommended to patients regardless of the practice setting of the physician; meanwhile, weight loss was more frequently recommended by GEs than by PCPs.

Several studies have demonstrated that there is often poor agreement between patients and physicians in their assessment of GERD symptom severity, with physicians tending to underestimate symptom severity and the impact on healthrelated quality of life, which is an essential component of providing proper medical care. ${ }^{71,72}$ As the role for PCPs in the management of GERD continues to evolve and expand, there is a big challenge for PCPs to improve clinical outcomes and patient satisfaction by strengthening physician-patient relationships. A better understanding of each patient's experience of the disease will help PCPs to appreciate that even mild symptoms of GERD can be troublesome and can be associated with a clinical reduction in patient well-being. Consequently, a need exists for improved questioning during 
consultation and more effective communication to assist in eliciting the most relevant information from patients. It is suggested that this process can be augmented by the use of relevant patient-reported management and outcome instruments, which can facilitate patient communication and help physicians understand and satisfy the needs of patients with GERD. ${ }^{73,74}$ PCPs should also consider that issues related to dosing and treatment adherence (compliance) may be involved when an incomplete response to PPI therapy is apparent and well-being continues to be impaired. Once the physician has confirmed that GERD symptoms are still present during PPI therapy, the challenge will be to determine whether treatment is being taken as prescribed. For example, the importance of treatment adherence and ingestion of PPI therapy before a meal should be stressed.

\section{Inflammatory bowel disease}

The fact that inflammatory bowel disease (IBD) is a chronic disease, affecting primarily young adults for the rest of their life, results in a huge impact on health services because these patients require life-lasting medical care as well as clinical and laboratory investigation. ${ }^{75-77}$ A significant part of these services refer to primary care, in which the PCP plays a key role. For PCPs, both early diagnosis and proper treatment represent a real challenge regarding their effort to ensure the best possible quality of life in patients with IBD.

Early diagnosis of IBD represents an important factor related to favorable response to treatment. Because accurate diagnosis of ulcerative colitis and Crohn's disease (CD) is mainly based on endoscopic and histological examinations, which are usually performed by GEs, PCPs should mainly focus on the details of the clinical picture. Diarrhea is the predominant symptom in both clinical situations; the diagnosis should be considered as possible in any patient presenting with diarrhea that persists for more than 2 weeks. Diarrhea, depending on the location and extent of disease, may be accompanied by other gastrointestinal or systemic symptoms, the exact assessment of which will greatly facilitate the differential diagnosis from other situations such as infectious colitis, celiac disease, irritable bowel syndrome (IBS), diverticular disease, etc. Apart from diarrhea, the characteristics of abdominal pain should be carefully evaluated as this symptom often accompanies the onset of IBD. Specifically, in ulcerative colitis, pain is usually mild and physical examination usually reveals tenderness in the left iliac fossa in the suprapubic area. In $\mathrm{CD}$, pain is usually located in the right lower abdomen with or without an accompanied palpable mass - a finding that could be difficult to be differentiated from an attack of acute appendicitis. Apart from the usual location of pain, pain is diffused in other cases and may be accompanied by bloating, abdominal distension, and nausea or vomiting. In these cases, the presence of blood in the stool could substantially contribute to the effort to exclude the diagnosis of IBS, which can sometimes be manifested with a similar clinical picture. ${ }^{77,78}$ It is particularly important for the PCP to be able to recognize anal disease (fleshy skin tags, fissures, fistulas) and oral manifestations (mouth ulcers, swollen lips), on the basis of which one can make the correct clinical diagnosis of CD. Particular attention should be given to the recognition of extraintestinal manifestations of IBD, such as arthralgias, uveitis, and erythema nodosum, the presence of which could increase the possibility of early diagnosis, thus avoiding unnecessary referrals to other specialties.

Dealing properly with patients with IBD and achieving the best possible quality of life requires a multidisciplinary approach with many key players, involving PCPs, GEs, surgeons, radiologists, pathologists, psychologists, rheumatologists, and dietitians. Only by taking a long-term approach in treatment decisions, delivering a patient-centered multidisciplinary approach, and adopting a chronic disease pathway to management will an optimal outlook for the vast majority of patients with IBD be achieved. ${ }^{79}$ However, the majority of patients with IBD are given follow-up in a specialized gastroenterology department of a hospital, and only a small proportion of them are referred to PCPs for medical care. ${ }^{80}$ This leads to significant shortcomings such as difficult access, increased costs, and unnecessary involvement of the specialist, mainly due to the lack of relevant information from patients and their family. These weaknesses can be overcome with the active involvement of the PCP, who will contribute significantly to the continuous, coordinated, and holistic health care for patients with IBD. This is supported by available data showing that satisfaction with the primary physician did not depend on physician type (for example, GE versus general practitioner). ${ }^{81,82}$ However, it should be emphasized that data concerning the degree of involvement of PCPs in management of IBD patients are limited.

A GE usually does the initial administration of drugs in IBD. However, the PCP is responsible for monitoring the compliance of the patient to treatment and, if necessary, for making dose adjustments in close cooperation with the specialist. The PCP must be able to recognize early any adverse effects of aminosalicylates, corticosteroids, or immunosuppressive drugs used in IBD. Particular attention must be given to the prevention and treatment of osteoporosis (eg, calcium and vitamin D supplementation) in people 
who receive corticosteroids for a long period. Also, patients receiving immunosuppressives such as azathioprine and 6-mercaptopurine, as well as immunomodulators such as infliximab and adalimumab, should be subjected to regular blood examinations, including full blood count every week for the first month, every 2 weeks for next 1 month, and then every month.

During the follow-up period, the PCP should be alert in order to recognize early outbreaks of the disease and to identify factors that contribute to the appearance of recurrence. The PCP must be available at any time and, when necessary, must refer the patient immediately to the specialist. Moreover, open access to the specialists seems to be preferred by the patients with IBD as well as by the PCP. ${ }^{83}$ On the other hand, the PCP acting as a gatekeeper must avoid unnecessary referrals, which enhance the work of specialists and the secondary care services. The PCP must be aware of the need for prompt medical attention and investigation if the suspicion of infection arises while on therapy with biologic agents. The combination of drugs, particularly where steroids are used in moderate or high doses, carries the greatest risk of severe infection. Given the risks of infection, vaccination and screening have become an important issue in IBD. This includes screening for tuberculosis and hepatitis B infection before initiating therapy, checking immunization/past infection status of relevant viral diseases (eg, varicella zoster), ensuring vaccination for influenza and pneumococcus, and advice regarding travel (eg, avoid travelling to areas where tuberculosis is endemic if taking an anti-tumor-necrosisfactor agent). ${ }^{79}$

One important factor that contributes to the improvement of quality of life of patients with IBD is education regarding features of the disease, the need for the involvement of many disciplines to tackle the disease, and the procedures needed to be followed. ${ }^{84}$ At the same time, the patient should be informed and also be open to any questions and concerns that are often presented, such as what possibilities there are of exacerbation of the disease or of developing cancer and whether the disease will affect their sexual life or reproductive capacity. The IBD can affect seriously the self-image of patients, their ability to work, and interpersonal, family, and social relationships. Many times, patients with IBD have a changing emotional state - similar to those suffering from incurable disease - which begins with denial, followed by anger, negotiation, sadness, and, finally, acceptance of the disease. Therefore, the treatment should focus not only on the disease activity, but also on psychosocial problems such as anxiety, depression, and occupational, social, and sexual rejection, which the PCP should recognize early and address. $^{85}$

\section{Irritable bowel syndrome}

IBS is a global problem and is more common in women than in men. The prevalence of IBS ranges from $3 \%-25 \%$ of adults and causes much morbidity and cost in terms of lost time at work and drain on health resources. ${ }^{86}$ Because the symptoms of IBS are common to a number of other GI conditions, IBS was long considered a "diagnosis of exclusion", leading to excessive testing of patients with characteristic symptoms. Fortunately, advances in research have led to the development of symptom-based approaches, aimed at standardizing IBS patient subgroups, and the development of consensus guidelines advocating a positive diagnosis of IBS based primarily on the pattern and nature of symptoms, without the need for excessive laboratory testing. ${ }^{87,88}$

Properly diagnosing IBS can be challenging and uncertain for several reasons, including that: IBS has no consistent biological marker, which leaves physicians relying on patient symptoms alone to make the diagnosis; the symptoms of IBS are often difficult to quantify objectively; and many organic conditions can masquerade as IBS. The last fact is the most troubling to both physicians and patients, many of whom are unsettled by the prospect that alternative diagnoses have been overlooked (such as IBD, microscopic colitis, infectious colitis, small intestinal bacterial overgrowth, celiac sprue, and colon neoplasia). This uncertainty often prompts clinicians to approach IBS as a diagnosis of exclusion by performing tests to exclude alternative etiologies. ${ }^{89}$ Conversely, the Rome III guidelines state that IBS can be diagnosed in the absence of "alarm features", and is "often properly diagnosed without testing", encouraging clinicians to make a positive diagnosis of IBS on the basis of validated symptom criteria, and emphasizing that IBS is not a diagnosis of exclusion. ${ }^{90}$

However, the reality is different. Most PCPs still believe IBS is a diagnosis of exclusion, and best-practice diagnostic guidelines have not been uniformly adopted. A recent study of Spiegel et $\mathrm{al}^{91}$ showed that only $8 \%$ of experts endorsed IBS as a diagnosis of exclusion, whereas $72 \%$ of community providers shared this belief. Moreover, they found that providers who believe IBS is a diagnosis of exclusion ordered more diagnostic tests and consumed between $\$ 200$ and \$400 more in diagnostic expenditures per patient compared with those who did not share this belief. Using a standardized definition of appropriateness, the study showed that experts only rated celiac sprue screening and a complete blood count as generally 
appropriate first-line routine tests in diarrhea-predominant IBS (D-IBS), whereas, in a 52-year-old constipation-predominant IBS (C-IBS) patient, only complete blood count and colonoscopy were considered as appropriate by experts. In contrast, community providers rated complete blood count, chemistry panel, stool leukocytes, and stool ova and parasites as appropriate in D-IBS, whereas, in C-IBS, these providers rated serum chemistry panel, colonoscopy, complete blood count, and thyroid-stimulating hormone level as appropriate. These findings suggest that frontline providers are especially prone to order a wide variety of tests with low diagnostic yield that may have significant economic implications.

The aforedescribed situation probably reflects the level of awareness and knowledge of IBS guidelines among PCPs. Although those guidelines have been available for more than two decades, available data show that IBS criteria are largely unknown and are poorly validated in general practice where most patients are treated. ${ }^{50,92,93}$ An early UK study from 2003 showed that only $21 \%$ of general practitioners had heard of the Manning criteria and $12 \%$ had heard of the Rome criteria for the diagnosis of functional GI disorders, compared with $81 \%$ and $83 \%$, respectively, of hospital specialists (GEs and surgeons). ${ }^{92}$ This figure seems to be similar to a later study (2008), where 23\% of European PCPs were familiar with any of the diagnostic criteria, and, of those, only $20 \%$ actually used these criteria in their clinical practice. ${ }^{50}$ Moreover, these findings are in line with those of a recent study from 2012 that demonstrated that only $10 \%$ of Iceland PCPs had heard of the Manning criteria, 27\% had heard of Rome I, and $17 \%$ had heard of Rome II. In contrast, more than $80 \%$ of GEs reported that they were knowledgeable about those $^{\text {criteria. }}{ }^{93}$

Treatment of IBS relies on a positive diagnosis, reassurance, lifestyle advice, and pharmacological and psychological therapies. ${ }^{87,88}$ However, many patients suffer ongoing symptoms, and a small proportion of IBS patients were satisfied with the treatment they had been given. ${ }^{92,93}$ Bulking agents and antispasmodics are the most commonly prescribed medications. Olafsdottir et $\mathrm{al}^{93}$ observed that both PCPs and GEs gave their patients mebeverine in most cases. Psyllium was frequently used by GEs, chlordiazepoxide and clidinium was used more often by PCPs, and antidepressants were used in some cases, especially by GEs. Although the evidence supports rifaximin as an emerging treatment for IBS, so far, there are no published data about the prescription patterns of rifaximin in clinical practice. IBS guidelines have highlighted the lack of evidence for the current drug management, but still recommend them as first line management in primary care. ${ }^{94}$
However, it has been accepted that medications are largely ineffective in symptom management, and physicians are expected to design a long-term and nonpharmacological approach to help the patient adjust to their chronic illness. From that point of view, the expanding role of PCPs in nonpharmacological interventions, such as diet, will contribute to the increase of standardization in clinical practice.

Current evidence-based guidelines for the dietary management of IBS recommend, first of all, that, before any dietary intervention, it may be useful to ask individuals to keep a food and symptom diary. ${ }^{95}$ In particular, the frequency and timing of symptoms (eg, meal-related, daily, nocturnal, weekdays, weekends, holidays, exercise induced, and, for women only, whether symptoms are related to their menstrual cycle, gut hypersensitivity) should be considered. Basic dietary instruction for patients with IBS should include advice to eat regularly, eat meals slowly, and drink 1.5-3 L of caffeine- and alcohol-free noncarbonated liquids per day. Second-line dietary management includes advanced dietary interventions to alleviate symptoms resulting from nonstarch polysaccharides and fermentable carbohydrates. The available evidence suggests that linseeds may be effective for C-IBS type. In contrast, wheat bran does not relieve IBS symptoms. Fructose, sorbitol, and galacto-oligosaccharides can worsen bloating in IBS, and elimination or empiric diets are considered third-line dietary therapy for IBS..$^{95}$ The use of probiotics in patients with IBS is an area of emerging interest, and many GEs recommend probiotics. PCPs are increasingly confronted with questions about the suitability (or otherwise) of probiotics, but their familiarity with probiotics is limited. At the same time, the public is exposed to widespread claims for probiotics with a variety of products in shops. The randomized placebo-controlled trials included in a recent analysis support, with a high evidence level, a role for specific probiotics in the management of overall symptoms and abdominal pain in patients with IBS, and for preventing or reducing diarrhea in patients receiving antibiotics or $H$. pylori eradication triple therapy. ${ }^{96}$ In practice, this means that probiotics with supportive evidence for benefit should be tried. However, the need for more objective evidence-based guidance on the role of probiotics is becoming increasingly important as public awareness of probiotics grows as a result of considerable media interest and intensive advertising campaigns.

Functional GI diseases are chronic conditions that require long-term management of symptoms, which can often lead to frustration both on the part of the patient and the physician. As such, the role of the therapeutic physician-patient rela- 
tionship in diagnosis and management of IBS has been attracting increasing attention. A patient-centered approach with a strong focus on effective communication between the physician and the patient has been recommended for management of functional bowel diseases such as IBS and has been associated with improved outcomes, increased patient satisfaction, and decreased utilization of health care. ${ }^{97}$ For these reasons, a strong therapeutic relationship between the PCP and the patient constitutes an instrumental component in the successful management of functional GI disorders. Relevant guidelines recommend a multistep process focused on a patient-centered approach to obtain the patient history, careful examination, patient-focused education, setting realistic expectations for therapy, involvement of the patient in treatment decisions, and establishing a long-term relationship. ${ }^{98}$

\section{Other gastrointestinal disorders}

Celiac disease $(\mathrm{CeD})$ represents an under-estimated and under-diagnosed disease in primary care. The prevalence of celiac disease is estimated to be $0.8 \%-1.9 \%$ in the general population, and $4.5 \%-12 \%$ among first-degree relatives. The delay in the final diagnosis is quite large; studies show an average of more than 10 years from symptom onset to diagnosis. ${ }^{99,100}$ Undiagnosed CeD increases the risk of early mortality and associated autoimmune disease and can lead to neurologic complications, osteoporosis, and gastrointestinal malignancy. PCPs must cultivate a high index of suspicion for $\mathrm{CeD}$ and bear it in mind as a differential diagnosis in many clinical situations. Patients with signs or symptoms indicating $\mathrm{CeD}$ (chronic or intermittent diarrhea, persistent or unexplained GI symptoms such as nausea and vomiting, prolonged fatigue, recurrent abdominal pain, cramping or distension, sudden/unexpected weight loss, and unexplained iron-deficiency anemia) should be subjected to serological test for immunoglobulin A anti-tissue transglutaminase antibody. Also, information concerning autoimmune thyroid disease, dermatitis herpetiformis, IBS, type 1 diabetes, or existence of first-degree relatives with $\mathrm{CeD}$ should be considered. If the serological test is positive, a gastroscopy with duodenal biopsies should be carried out.

Chronic constipation (CC) is a common disorder that presents with a prevalence of $16 \%$ in adults overall and $33 \%$ in those older than 60 years. Although physicians often regard constipation as being synonymous with infrequent bowel movements, typically fewer than three per week, patients have a broader set of symptoms, including hard stools, a feeling of incomplete evacuation, abdominal discomfort, bloating, and distention, as well as other symptoms (ie, excessive straining, a sense of anorectal blockage during defecation, and the need for manual maneuvers during defecation), which suggest a defecatory disorder. ${ }^{101}$ Patients with chronic idiopathic constipation have a range of colonic motor disorders. The majority $(80 \%)$ have slow transit constipation, dyssynergic defecation, or a combination of slow transit constipation and dyssynergic defecation. In addition, many patients (51\%) with chronic idiopathic constipation have a concurrent upper GI tract transit disorder. ${ }^{102}$

After the initial history and physical examination, in the absence of other symptoms and signs, only a complete blood cell count is necessary. Although metabolic tests (thyroid-stimulating hormone, serum glucose, creatinine, and calcium) are often performed, their diagnostic utility and cost-effectiveness have not been rigorously evaluated and are probably low. Colonoscopy should be performed in patients with alarm features (ie, blood in stools, anemia, weight loss, significant abdominal pain, family history of CRC or IBD) and in those over 50 years to screen for CRC. ${ }^{94}$

The treatment of CC involves the use of dietary fiber, bulking agents, osmotic and stimulant laxatives, stool softeners, prokinetics, biofeedback training, and surgery. Despite the wide range of therapeutic options, almost half of affected patients report a lack of complete relief from their symptoms. ${ }^{103}$ Trials conducted in primary care patients are lacking for all therapies. In order to improve the management of ambulatory patients with CC, Pare ${ }^{103}$ suggests a practical management algorithm using a multistep approach favoring early introduction of combined therapies and a long-term step-down strategy to the lowest satisfactory regimen.

Fecal incontinence (FI) is a devastating disorder that often is under-reported by patients and under-detected by physicians. Many factors contribute to the pathophysiology of FI, including advanced age, bowel irregularity, parity, and obesity. ${ }^{104}$ FI is more common than previously thought, with a prevalence that varies by the population studied. In a self-reported survey, $36.5 \%$ of primary care patients reported episodes of FI, but only $2.7 \%$ of these patients had a documented diagnosis, ${ }^{105}$ suggesting that PCPs could play a vital role in FI diagnosis. A detailed history and focused rectal examination are important to making the diagnosis and determining contributing causes. Although multiple diagnostic studies are available to assess the cause of FI, specific guidelines that delineate when testing should be done do not exist. All types of FI are initially managed in the same way, which includes lifestyle modification to reduce bowel derangements, improved access to toileting, and initiation 
of a bulking regimen to improve stool consistency. If initial conservative management fails, pharmacologic agents, biofeedback, or surgery may be indicated.

\section{Conclusion}

It is well known that GI disorders are common in general practice, and the PCP has a central role in the diagnosis and management of those problems in the primary care setting. Although national and international appropriate evidencebased standards are available, a considerable number of PCPs do not routinely follow them. This review demonstrates a wide range of awareness and application of guidelines concerning GI disorders among PCPs worldwide. In relation to this, it has been argued that PCPs constitute a very heterogeneous group with respect to qualification and special interest, and the ability to meet expectations of modern medicine varies widely from country to country, probably because of significant differences in the national health systems and continuous education programs. ${ }^{50}$ Moreover, it could be noted that the differences in guidelines for GI diseases and perceptions about importance of primary health care that exist between various countries probably influence the worldwide discrepancies in the practice of PCPs.

The present review also refers to the differences in knowledge, attitudes, and patterns of care between PCPs and specialists (GEs). It has been reported that GEs were generally more knowledgeable in terms of widely accepted standards of care (including clinical guidelines), were using a more resourceintensive approach, and were quicker in adoption of new and effective treatments than PCPs. ${ }^{51-53,69,92,93}$ It seems that this pattern of differences between generalists and specialists is common in many other disease areas. ${ }^{106}$ Meanwhile, it should be noted that these findings are derived from physician-based surveys and there is a lack of information about the impact of these differences in outcomes, related cost, and in patient psychosocial well-being and satisfaction.

The critical role of PCPs in GI cancer screening and early detection is well established. The presented evidence indicates that primary care plays an important role in increasing CRC screening rates because the recommendation of PCP is one of the strongest predictors of adherence to CRC screening. ${ }^{4,6,11,12,107}$ Our review highlights the modern medicine request that all adults of $>50$ years should be considered for screening for CRC in the same way that women are routinely screened for breast or cervical cancer; the age for testing and the type of test needs to be tailored to individual patients. Therefore, further positive engagement of PCPs with $\mathrm{CRC}$ screening (and with FOBt in particular) is required in order to reach acceptable levels in screening rates. Some evidence shows that higher $\mathrm{CRC}$ rates may be achieved with involvement of nonphysician health care providers (including nurse practitioners and physician assistants), suggesting that it is very essential for PCPs to develop interactions with community professionals and support teamwork incentives within the health care system. ${ }^{108,109}$

On the other hand, the identification of high-risk patients and prompt endoscopic examination are the best strategies for early diagnosis of upper GI malignancies. It is worth noting that, despite advances in diagnostic technology, an accurate and complete medical history (including family history) in combination with detailed physical examination remains the most critical aspect of diagnosing GI malignancies and other serious diseases such as IBD. Taking into account evidence indicating suboptimal practices of PCPs in confronting patients with alarm features such as iron-deficient anemia, ${ }^{27,28}$ we underscore the need for complete endoscopic investigation and long-term follow-up when no cause is detected in those patients. ${ }^{26}$

Dyspepsia and GERD represent the most common benign GI disorders in clinical practice. The discovery of $H$. pylori and PPIs has revolutionized the clinical approach and treatment of those conditions. Guidelines regarding dyspepsia and H. pylori infection, which have been disseminated for more than 15 years, ${ }^{42,46}$ recommend that most patients with recurrent dyspeptic symptoms should be tested and treated for $H$. pylori. However, there appears to be under- and inappropriate treatment of $H$. pylori infection in primary care and a low rate of retesting after eradication, indicating that guidelines are not well implemented in practice. ${ }^{47-53}$ Since the role for PCPs in the management of GERD continues to evolve and expand, there is increasing potential for PCPs to improve the value of their care services for GERD patients. This can be reached with better understanding of patients' needs, effective lifestyle interventions, and appropriate use of diagnostic and therapeutic tools. As mentioned before, inappropriate prescription of PPIs is a matter of concern. ${ }^{55-61}$ Bearing in mind that the unnecessary use of PPIs could cause potential harm (including pneumonia, fracture, enteric infection, and malabsorption) and pharmacological interactions, and could lead to polypharmacy, especially in older adults, PCPs play a pivotal role in the attempt to rationalize the use of PPIs and to reduce expenditure. We suggest that educational programs that focus on optimal management of $H$. pylori infection in parallel with appropriate use of PPIs would be very helpful in improving the quality of care in patients with upper GI symptoms. 
IBD is a complicated disease and early diagnosis represents a real challenge for PCPs. Monitoring patients with IBD is also an important role assumed by PCPs. In order to respond adequately to these challenges, PCPs are invited to demonstrate their diagnostic skills in early detection of relapses, their ability to provide continuous supporting therapies, and their ability to coordinate patient care as given by a multidisciplinary team.

IBS is the most complex functional GI disorder and constitutes a dynamic field characterized by significant changes in diagnostic strategies and therapeutic options over the last decade. ${ }^{110}$ Evidence-based guidelines suggest that IBS could be confidently diagnosed in the clinic at the time of the first visit using the Rome criteria and a careful history and physical examination. However, data show that PCPs are unfamiliar with recommended diagnostic criteria, and the majority of them still believe that IBS is a "diagnosis of exclusion". ${ }^{50,92,93}$ Effective educational interventions are needed to update the perceptions of PCPs regarding IBS and to overcome the inertia of previous practice. Treatment options for IBS have increased in number in the past decade and clinicians should not be limited to using only fiber supplements and antispasmodic drugs. As we mentioned, the expanding role of PCPs in nonpharmacological interventions such as diet will contribute to the increase of standardization in clinical practice. The diet merits special attention, particularly in cases of patients with IBS and comorbidities such as diabetes, obesity, dyslipidemia, gallstones, renal failure, and oral anticoagulants treatment, where PCPs are calling to dissolve the confusion that exist with regard to what foods are and are not allowed.

It is obvious that PCPs, when dealing with chronic GI disorders, face a set of complex challenges related to the needs of their patients on the one side, and the support of the health care system that they are serving on the other side. This is a difficult balancing act that can be achieved only via the conscious dedication of PCPs to patients' problems and the willingness of PCPs to continuously updated their knowledge and skills. Also, an improvement in the quality and concurrent cost-effectiveness of care can only be achieved through well-educated PCPs.

PCPs have a duty to keep their professional practices and skills up-to-date, which can be accomplished by means of continuing medical education. First of all, they must be familiar with literature concerning national or regional (eg, North American, European, Asia Pacific) guidelines and must have the ability of critical reading; these skills should be cultivated from residency and perfected during the following years in practice. Because PCPs have to choose from guidelines that are numerous, not always specifically addressed to them, sometimes differing, and occasionally contradictory, every effort should be made to assess the quality of the guideline as a whole, determine its currency, and assess the content of the recommendations before deciding to adopt and tailor to a local context. ${ }^{111}$ However, sufficient knowledge of guidelines is not enough; further educational efforts are needed for appropriate implementation into clinical practice. Continuing medical education has traditionally employed conferences, courses, symposia, workshops, and small-group discussions as the most common methods for physicians to maintain their competence. The rapid development of internet-based communications has facilitated the identification of useful evidence and has allowed for new online self-learning opportunities for physicians, but this has not yet replaced the face-to-face teaching. There is enough evidence to show that the most effective educational interventions for primary care professionals to promote the early diagnosis of cancer (including colon, breast, skin, cervical, and prostate cancer) are interactive education, computerized reminder systems, audit, and feedback, ${ }^{112}$ whilst didactic teaching as a single method seems to be ineffectual in improving cancer screening rates and also in changing attitudes of PCPs concerning management of chronic diseases (eg, IBS). ${ }^{12,113}$ Moreover, it is well recognized that educational programs or strategies that involve two or more interventions appear to be more effective than single interventions, particularly when measures to enhance knowledge and awareness are combined with interventions to facilitate and reinforce performance in everyday practice. ${ }^{114}$ In addition, we strongly suggest that periodic onjob training courses (eg, every 10 years) in gastroenterology clinics constitute a very effective method for updating PCPs' diagnostic and therapeutic skills.

\section{Disclosure}

The authors report no conflicts of interests in this work.

\section{References}

1. Peery AF, Dellon ES, Lund J, et al. Burden of gastrointestinal disease in the United States: 2012 update. Gastroenterology. 2012;143:1179-1187.

2. Jones RH. Primary care research and clinical practice: gastroenterology. Postgrad Med J. 2008;84:454-458.

3. Flook NW. Management of gastrointestinal disease: Returning it to primary care. Can Fam Physician. 2004;50:685-686. [English, French].

4. Fletcher RH. Successful colorectal cancer screening starts with primary care. Rev Gastrointerol Disord. 2002;2 Suppl 1:S27-S34.

5. Seeff LC, Nadel MR, Klabunde CN, et al. Patterns and predictors of colorectal cancer test use in the adult US population. Cancer. 2004;100: 2093-2103. 
6. Hanks H, Veitch C, Harris M. Colorectal cancer management - the role of the GP. Aust Fam Physician. 2008;37:259-261.

7. Schattner A, Gilad A. Primary care physicians' awareness and implementation of screening guidelines for colorectal cancer. Prev Med. 2002;35:447-452.

8. Xilomenos A, Mauri D, Kamposioras K, et al. Colorectal cancer screening awareness among physicians in Greece. BMC Gastroenterol. 2006;6:18

9. Mauri D, Pentheroudakis G, Milousis A, et al. Colorectal cancer screening awareness in European primary care. Cancer Detect Prev. 2006;30:75-82.

10. Allemani C, Rachet B, Weir HK, et al. Colorectal cancer survival in the USA and Europe: a CONCORD high-resolution study. BMJ Open. 2013;3(9):e003055.

11. Cole SR, Young GP, Byrne D, Guy JR, Morsom J. Participation in screening for colorectal cancer based on fecal occult blood test is improved by endorsement by the primary care practitioner. $J \mathrm{Med}$ Screen. 2002;9:147-152.

12. Davis TC, Arnold CL, Rademaker AW, et al. FOBT completion in FQHCs: impact of physician recommendation, FOBT information, or receipt of the FOBT kit. J Rural Health. 2012;28:306-311.

13. Panzuto F, Chiriatti A, Bevilacqua S, et al. Symptom-based approach to colorectal cancer: survey of primary care physicians in Italy. Dig Liver Dis. 2003;35:869-875.

14. Astin M, Griffin T, Neal RD, Rose P, Hamilton W. The diagnostic value of symptoms for colorectal cancer in primary care: a systematic review. Br J Gen Pract. 2011;61:e231-e243.

15. Achkar E. Colorectal cancer screening in primary care: the long and short of it. Am J Gastroenterol. 2004;99:837-838.

16. Seeff LC, Richards TB, Shapiro JA, et al. How many endoscopies are performed for colorectal cancer screening? Results from CDC's survey of endoscopic capacity. Gastroenterology. 2004;127:1670-1677.

17. Edwards JK, Norris TE. Colonoscopy in rural communities: can family physicians perform the procedure with safe and efficacious results? J Am Board Fam Pract. 2004;17:353-358.

18. Wilkins T, LeClair B, Smolkin M, et al. Screening colonoscopies by primary care physicians: a meta-analysis. Ann Fam Med. 2009;7: 56-62.

19. Azzopardi J, DeWitt DE. Quality and safety issues in procedural rural practice: a prospective evaluation of current quality and safety guidelines in 3000 colonoscopies. Rural Remote Health. 2012;12:1949.

20. Kolber MR, Wong CK, Fedorak RN, Rowe BH; on behalf of the APC-Endo Study Physicians. Prospective Study of the Quality of Colonoscopies Performed by Primary Care Physicians: The Alberta Primary Care Endoscopy (APC-Endo) Study. PLoS One. 2013;8:e67017.

21. Yuan Y. A survey and evaluation of population-based screening for gastric cancer. Cancer Biol Med. 2013;10:72-80.

22. Asaka M, Kato M, Graham DY. Strategy for eliminating gastric cancer in Japan. Helicobacter. 2010;15:486-490.

23. Dan YY, So JB, Yeoh KG. Endoscopic screening for gastric cancer. Clin Gastroenterol Hepatol. 2006;4:709-716.

24. Ryan J, Murkies A. Diagnosis of upper gastrointestinal malignancy. Aust Fam Physician. 2006;35:200-201.

25. Maconi G, Manes G, Porro GB. Role of symptoms in diagnosis and outcome of gastric cancer. World J Gastroenterol. 2008;14:1149-1155.

26. Goddard AF, James MW, McIntyre AS, Scott BB; British Society of Gastroenterology. Guidelines for the management of iron deficiency anaemia. Gut. 2011;60:1309-1316.

27. Logan EC, Yates JM, Stewart RM, Fielding K, Kendrick D. Investigation and management of iron deficiency anaemia in general practice: a cluster randomized controlled trial of a simple management prompt. Postgrad Med J. 2002;78:533-537.

28. Droogendijk J, Beukers R, Berendes PB, Tax MG, Sonneveld P, Levin MD. Screening for gastrointestinal malignancy in patients with iron deficiency anemia by general practitioners: an observational study. Scand J Gastroenterol. 2011;46:1105-1110.
29. Bramble MG, Suvacovic Z, Hungin AP. Detection of upper gastrointestinal cancer in patients taking antisecretory therapy prior to gastroscopy. Gut. 2000;46:464-467.

30. Panter SJ, O'Flanagan H, Bramble MG, Hungin AP. Empirical use of antisecretory drug therapy delays diagnosis of upper gastrointestinal adenocarcinoma but does not effect outcome. Aliment Pharmacol Ther. 2004;19:981-988

31. Wayman J, Hayes N, Raimes SA, Griffin SM. Prescription of proton pump inhibitors before endoscopy. A potential cause of missed diagnosis of early gastric cancers. Arch Fam Med. 2000;9:385-388.

32. Voutilaimen M, Kunnamo I. A survey of open-access endoscopy in primary health care centers: outcome of gastric carcinoma patients diagnosed by general practitioners compared with hospital-referred endoscopy. Dig Liver Dis. 2005;37:119-123.

33. Lagergren J, Lagergren P. Oesophageal cancer. BMJ. 2010;341:c6280.

34. Witzig R, Schönberger B, Fink U, et al. Delays in diagnosis and therapy of gastric cancer and esophageal adenocarcinoma. Endoscopy. 2006;38:1122-1126.

35. Subasinghe D, Samarasekera DN. Delay in the diagnosis of esophageal carcinoma: experience of a single unit from a developing country. Indian $J$ Cancer. 2010;47:151-155.

36. Hippisley-Cox J, Coupland C. Identifying patients with suspected gastro-oesophageal cancer in primary care: derivation and validation of an algorithm. Br J Gen Pract. 2011;61:e707-e714.

37. Collins GS, Altman DG. Identifying patients with undetected gastrooesophageal cancer in primary care: External validation of QCancer ${ }^{\mathbb{B}}$ (Gastro-Oesophageal). Eur J Cancer. 2013;49:1040-1048.

38. Fitzgerald RC, di Pietro M, Ragunath K, et al. British Society of Gastroenterology guidelines on the diagnosis and management of Barrett's oesophagus. Gut. 2014;63:7-42.

39. Ford AC, Moayyedi P. Dyspepsia. BMJ. 2013;347:29-33.

40. Hungin AP, Rubin GP. Management of dyspepsia across the primarysecondary healthcare interface. Dig Dis. 2001;19:219-224.

41. Ford AC, Marwaha A, Lim A, Moayyedi P. What is the prevalence of clinically significant endoscopic findings in subjects with dyspepsia? Systematic review and meta-analysis. Clin Gastroenterol Hepatol. 2010;8:830-837.

42. Malfertheiner P, Megraud F, O'Morain CA, et al. The European Helicobacter Study Group (EHSG). Management of Helicobacter pylori infection - the Maastricht IV/Florence consensus report. Gut. 2012;61(5):646-664.

43. Vakil N. Dyspepsia, peptic ulcer, and H. pylori: a remembrance of things past. Am J Gastroenterol. 2010;105:572-574.

44. Wee EW. Evidence-based approach to dyspepsia: from Helicobacter pylori to functional disease. Postgrad Med. 2013;125:169-180.

45. Talley NJ. Modern management of dyspepsia. Aust Fam Physician. 1996;25:47-52.

46. No authors listed. Current European concepts in the management of Helicobacter pylori infection. The Maastricht Consensus Report. European Helicobacter Pylori Study Group. Gut. 1997;41: $8-13$.

47. Bennett K, Feely J, Thornton O, Dobson M, O'Morain CA, O'Connor HJ. Impact of Helicobacter pylori on the management of dyspepsia in primary care. Aliment Pharmacol Ther. 2006;24:637-641.

48. Kim BG, Kim JW, Jeong JB, et al. Discrepancies between primary physician practice and treatment guidelines for Helicobacter pylori infection in Korea. World J Gastroenterol. 2006;12:66-69.

49. Ahmed S, Salih M, Jafri W, Ali Shah H, Hamid S. Helicobacter pylori infection: approach of primary care physicians in a developing country. BMC Gastroenterol. 2009;9:23.

50. Seifert B, Rubin G, de Wit N, et al. The management of common gastrointestinal disorders in general practice A survey by the European Society for Primary Care Gastroenterology (ESPCG) in six European countries. Dig Liver Dis. 2008;40:659-666.

51. O'Connor HJ. Helicobacter pylori and dyspepsia: physicians' attitudes, clinical practice, and prescribing habits. Aliment Pharmacol Ther. 2002;16:487-496. 
52. Hirth RA, Fendrick AM, Chernew ME. Specialist and generalist physicians' adoption of antibiotic therapy to eradicate Helicobacter pylori infection. Med Care. 1996;34:1199-1204.

53. Spiegel BM, Farid M, van Oijen MG, Laine L, Howden CW, Esrailian E. Adherence to best practice guidelines in dyspepsia: a survey comparing dyspepsia experts, community gastroenterologists and primary-care providers. Aliment Pharmacol Ther. 2009;29(8):871-881.

54. Chubineh S, Birk J. Proton pump inhibitors: the good, the bad, and the unwanted. South Med J. 2012;105:613-618.

55. Naunton M, Peterson GM, Bleasel MD. Overuse of proton pump inhibitors. J Clin Pharm Ther. 2000;25:333-340.

56. Batuwitage BT, Kingham JG, Morgan NE, Bartlett RL. Inappropriate prescribing of proton pump inhibitors in primary care. Postgrad Med J. 2007;83:66-68.

57. Heidelbaugh JJ, Kim AH, Chang R, Walker PC. Overutilization of proton-pump inhibitors: what the clinician needs to know. Therap $A d v$ Gastroenterol. 2012;5:219-232.

58. Patterson Burdsall D, Flores HC, Krueger J, et al. Use of proton pump inhibitors with lack of diagnostic indications in 22 Midwestern US skilled nursing facilities. J Am Med Dir Assoc. 2013;14:429-432.

59. Rostom A, Moayyedi P, Hunt R; Canadian Association of Gastroenterology Consensus Group. Canadian consensus guidelines on long-term nonsteroidal anti-inflammatory drug therapy and the need for gastroprotection: benefits versus risks. Aliment Pharmacol Ther. 2009;29:481-496.

60. Morini S, Zullo A, Oliveti D, et al. A very high rate of inappropriate use of gastroprotection for nonsteroidal anti-inflammatory drug therapy in primary care: a cross-sectional study. J Clin Gastroenterol. 2011;45: 780-784.

61. Zullo A, Hassan C, Oliveti D, et al. Helicobacter pylori management in non-steroidal anti-inflammatory drug therapy patients in primary care. Intern Emerg Med. 2012;7:331-335.

62. Katz PO, Gerson LB, Vela MF. Guidelines for the diagnosis and management of gastroesophageal reflux disease. Am J Gastroenterol. 2013;108:308-328.

63. Ornstein SM, Nietert PJ, Jenkins RG, Litvin CB. The prevalence of chronic diseases and multimorbidity in primary care practice: a PPRNet report. J Am Board Fam Med. 2013;26:518-524.

64. DeVault KR, Castell DO. Guidelines for the diagnosis and treatment of gastroesophageal reflux disease. Practice Parameters Committee of the American College of Gastroenterology. Arch Intern Med. 1995;15:2165-2173.

65. Jones R, Liker HR, Ducrotté P. Relationship between symptoms, subjective well-being and medication use in gastro-oesophageal reflux disease. Int J Clin Pract. 2007;61:1301-1307.

66. Gisbert JP, Cooper A, Karagiannis D, et al. Management of gastrooesophageal reflux disease in primary care: a European observational study. Curr Med Res Opin. 2009;25:2777-2784.

67. Wilcox CM, Heudebert G, Klapow J, Shewchuk R, Casebeer L. Survey of primary care physicians' approach to gastroesophageal reflux disease in elderly patients. J Gerontol A Biol Sci Med Sci. 2001;56:M514-M517.

68. Wong WM, Lim P, Wong BC. Clinical practice pattern of gastroenterologists, primary care physicians, and otolaryngologists for the management of GERD in the Asia Pacific region: the FAST survey. J Gastroenterol Hepatol. 2004;19:S54-S60.

69. Bretagne JF, Honnorat C, Richard-Molard B, Soufflet C, Barthélemy P. Perceptions and practices on the management of gastro-oesophageal reflux disease: results of a national survey comparing primary care physicians and gastroenterologists. Aliment Pharmacol Ther. 2007;25:823-833.

70. Halpern R, Kothari S, Fuldeore M, et al. GERD-related health care utilization, therapy, and reasons for transfer of GERD patients between primary care providers and gastroenterologists in a US managed care setting. Dig Dis Sci. 2010;55:328-337.

71. Fallone CA, Guyatt GH, Armstrong D, et al. Do physicians correctly assess patient symptom severity in gastro-oesophageal reflux disease? Aliment Pharmacol Ther. 2004;20:1161-1169.
72. McColl E, Junghard O, Wiklund I, Revicki DA. Assessing symptoms in gastroesophageal reflux disease: how well do clinicians' assessments agree with those of their patients? Am J Gastroenterol. 2005;100:11-18.

73. Flook NW, Wiklund I. Accounting for the effect of GERD symptoms on patients' health-related quality of life: supporting optimal disease management by primary care physicians. Int $J$ Clin Pract. 2007;61:2071-2078.

74. Ponce J, Garrigues V, Agréus L, et al. Structured management strategy based on the Gastro-oesophageal Reflux Disease (GERD) Questionnaire (GerdQ) vs usual primary care for GERD: pooled analysis of five cluster-randomised European studies. Int J Clin Pract. 2012;66:897-905.

75. de Boer AG, Sprangers MA, Bartelsman JF, de Haes HC. Predictors of health care utilization in patients with inflammatory bowel disease: a longitudinal study. Eur J Gastroenterol Hepatol. 1998;10:783-789.

76. Knutson D, Greenberg G, Cronau H. Management of Crohn's disease a practical approach. Am Fam Physician. 2003;68:707-714.

77. Gikas A. IBD and Primary Care Physician. In: Triantafillidis JK, Stanciu C, editors. Inflammatory Bowel Disease: Pathogenesis, Clinical Manifestations, Diagnosis, Treatment. Athens: Technogramma Publications; 2012:659-666.

78. Stotland BR, Stein RB, Lichtenstein GR. Advances in inflammatory bowel disease. Med Clin North Am. 2000;84:1107-1124.

79. Morrison G, Headon B, Gibson P. Update in inflammatory bowel disease. Aust Fam Physician. 2009;38:956-961.

80. Casellas F, Fontanet G, Borruel N, Malagelada JR. The opinion of patients with inflammatory bowel disease on healthcare received. Rev Esp Enferm Dig. 2004;96:174-184.

81. Hilsden RJ, Verhoef MJ, Best A, Pocobelli G. A national survey on the patterns of treatment of inflammatory bowel disease in Canada. BMC Gastroenterol. 2003;3:10.

82. Sossai P, Filippetti F, Muselmani AA, Catalini G. Inflammatory bowel diseases and the general practitioner's role in a region of Central Italy. Clin Exp Gastroenterol. 2010;3:27-31.

83. Williams JG, Cheung WY, Russell IT, Cohen DR, Longo M, Lervy B. Open access follow up for inflammatory bowel disease: pragmatic randomised trial and cost effectiveness study. BMJ. 2000;320(7234):544-548.

84. Husain A, Triadafilopoulos G. Communicating with patients with inflammatory bowel disease. Inflamm Bowel Dis. 2004;10:444-450.

85. Triantafillidis JK, Merikas E, Gikas A. Psychological factors and stress in inflammatory bowel disease. Expert Rev Gastroenterol Hepatol. 2013;7:225-238.

86. McFarland LV. State-of-the-art of irritable bowel syndrome and inflammatory bowel disease research in 2008. World J Gastroenterol. 2008;14:2625-2629.

87. Spiller R, Aziz Q, Creed F, Emmanuel A, Houghton L, Hungin P. Guidelines for the management of irritable bowel syndrome. Gut. 2007;56:1770-1798.

88. Irritable bowel syndrome in adults [webpage on the Internet]. National Institute for Health and Clinical Excellence; 2008. Available from: http:// www.nice.org.uk/CG061. Accessed October 11, 2013.

89. Spiegel BM. Do physicians follow evidence-based guidelines in the diagnostic work-up of IBS? Nat Clin Pract Gastroenterol Hepatol. 2007;4:296-297.

90. Longstreth GF, Thompson WG, Chey WD, Houghton LA, Mearin F, Spiller RC. Functional bowel disorders. Gastroenterology. 2006;130(5): 1480-1491.

91. Spiegel BM, Farid M, Esrailian E, Talley J, Chang L. Is irritable bowel syndrome a diagnosis of exclusion?: a survey of primary care providers, gastroenterologists, and IBS experts. Am J Gastroenterol. 2010;105: 848-858.

92. Gladman LM, Gorard DA. General practitioner and hospital specialist attitudes to functional gastrointestinal disorders. Aliment Pharmacol Ther. 2003;17:651-654.

93. Olafsdottir LB, Gudjonsson H, Jonsdottir HH, Jonsson JS, Bjornsson E, Thjodleifsson B. Irritable bowel syndrome: physicians' awareness and patients' experience. World J Gastroenterol. 2012;18:3715-3720. 
94. Saadi M, McCallum RW. Rifaximin in irritable bowel syndrome: rationale, evidence and clinical use. Ther Adv Chronic Dis. 2013;4: 71-75.

95. McKenzie YA, Alder A, Anderson W, et al; Gastroenterology Specialist Group of the British Dietetic Association. British Dietetic Association evidence-based guidelines for the dietary management of irritable bowel syndrome in adults. J Hum Nutr Diet. 2012;25:260-274.

96. Hungin AP, Mulligan C, Pot B, et al. Systematic review: probiotics in the management of lower gastrointestinal symptoms in clinical practice - an evidence-based international guide. Aliment Pharmacol Ther. 2013;38:864-886.

97. Di Palma JA, Herrera JL. The role of effective clinician-patient communication in the management of irritable bowel syndrome and chronic constipation. J Clin Gastroenterol. 2012;46:748-751.

98. Drossman DA. The functional gastrointestinal disorders and the Rome III process. Gastroenterology. 2006;130:1377-1390.

99. Michael M. Recognizing and managing celiac disease in primary care. J Am Acad Nurse Pract. 2003;15:108-114.

100. Khangura J, Van den Bruel A, Perera R, et al. Point-of-care testing for coeliac disease: primary care diagnostic technology update. $\mathrm{Br} J$ Gen Pract. 2013;63:e426-e428.

101. American Gastroenterological Association, Bharucha AE, Dorn SD, Lembo A, Pressman A. American Gastroenterological Association medical position statement on constipation. Gastroenterology. 2013;144:211-217.

102. Shahid S, Ramzan Z, Maurer AH, Parkman HP, Fisher RS. Chronic idiopathic constipation: more than a simple colonic transit disorder. J Clin Gastroenterol. 2012;46:150-154.

103. Pare P. The approach to diagnosis and treatment of chronic constipation: suggestions for a general practitioner. Can J Gastroenterol. 2011; 25 Suppl B:36B-40B.

104. Costilla VC, Foxx-Orenstein AE, Mayer AP, Crowell MD. Officebased management of fecal incontinence. Gastroenterol Hepatol (NY). 2013;9:423-433.
105. Dunivan GC, Heymen S, Palsson OS, et al. Fecal incontinence in primary care: prevalence, diagnosis, and health care utilization. $\mathrm{Am} \mathrm{J}$ Obstet Gynecol. 2010;202:493. e1-e6.

106. Harrold LR, Field TS, Gurwitz JH. Knowledge, patterns of care, and outcomes of care for generalists and specialists. J Gen Intern Med. 1999;14:499-511.

107. Ferrante JM, Lee JH, McCarthy EP, et al. Primary care utilization and colorectal cancer incidence and mortality among medicare beneficiaries: a population-based, case-control study. Ann Intern Med. 2013;159:437-446.

108. Hudson SV, Ohman-Strickland P, Cunningham R, Ferrante JM, Hahn K, Crabtree BF. The effects of teamwork and system support on colorectal cancer screening in primary care practices. Cancer Detect Prev. 2007;31:417-423.

109. Atassi K. Strategies to increase colorectal cancer screening. Nurse Pract. 2012;37:21-26.

110. Lacy BE, Weiser K, De Lee R. The treatment of irritable bowel syndrome. Therap Adv Gastroenterol. 2009;2:221-238.

111. Graham ID, Harrison MB. Evaluation and adaptation of clinical practice guidelines. Evid Based Nurs. 2005;8:68-72.

112. Schichtel M, Rose PW, Sellers C. Educational interventions for primary healthcare professionals to promote the early diagnosis of cancer: a systematic review. Educ Prim Care. 2013;24:274-290.

113. Longstreth GF, Burchette RJ. Family practitioners' attitudes and knowledge about irritable bowel syndrome: effect of a trial of physician education. Fam Pract. 2003;20:670-674.

114. Davis DA, Taylor-Vaisey A. Translating guidelines into practice. A systematic review of theoretic concepts, practical experience and research evidence in the adoption of clinical practice guidelines. CMAJ. 1997;157:408-416.
International Journal of General Medicine

\section{Publish your work in this journal}

The International Journal of General Medicine is an international, peer-reviewed open-access journal that focuses on general and internal medicine, pathogenesis, epidemiology, diagnosis, monitoring and treatment protocols. The journal is characterized by the rapid reporting of reviews, original research and clinical studies across all disease areas.

\section{Dovepress}

A key focus is the elucidation of disease processes and management protocols resulting in improved outcomes for the patient.The manuscript management system is completely online and includes a very quick and fair peer-review system. Visit http://www.dovepress.com/ testimonials.php to read real quotes from published authors. 